\title{
Role of Proton Pump Inhibitors on Rapid Urease Test (PYLO DRY) and Histopathological Examination
}

\author{
Manasa P Kumari ${ }^{1}$, Dr. M N Sumana ${ }^{2}$, Dr. Sunila ${ }^{3,}$ \\ Dr. Nanadeesh $\mathrm{H} \mathrm{P}^{4}$, Dr, Ardya $\mathrm{H} \mathrm{V}^{5}$ \\ ${ }^{1}$ Research Scholar, Department of Microbiology, JSS Medical College, Mysore \\ ${ }^{2}$ Professor of Microbiology, JSS Medical College, Mysore \\ ${ }^{3}$ Professor of Pathology, JSS Medical College, Mysore \\ ${ }^{4} \mathrm{HOD}$ and Professor of Gastroenterology, JSS Medical College, Mysore \\ ${ }^{5}$ Assistant Professor of Gastroenterology, JSS Medical College, Mysore
}

\begin{abstract}
In the diagnosis of Helicobacter pylori infection, rapid urease test (RUT) plays an important role. According to previous studies, if there was a low density of Helicobacter pylori in the stomach, then rapid urease test requires longer time to become positive. Density of Helicobacter pylori could be reduced by recent use of PPIs, hence rapid urease test may require longer reading time as well. The main objective was to determine the effect of PPI on the results of rapid urease test (PYLO DRY) and histopathology. Two antral biopsies were obtained from 35 dyspeptic patients, who underwent gastro intestinal endoscopy from March 2015 to July 2015. An informed consent from all patients and approval from the institutional ethical committee were obtained before proceeding. Antral biopsies were subjected for rapid urease test (PYLO DRY from HALIFAX RESEARCH LABORATORY KOLKATA, INDIA) and histopathologic examinations. The results of Rapid urease test were observed after 1 hour and 24 hours. The histopathologic examinations were used as the gold standard. PYLO DRY was positive in $17.6 \%$ (6) and $53 \%$ (18) when observed after one hour and 24 hours respectively. Eight were positive during histopathological examination. In our study, 24 hours Rapid urease test yeilded higher sensitivity but lower specificity compared to RUT at 1 hour for diagnosis of H.pylori infection in patients who were on PPI.
\end{abstract}

Keywords: Proton pump inhibitors (PPIs), Rapid Urease test (RUT), Helicobacter pylori, Antral biopsy

\section{Introduction}

Infection with H.pylori is worldwide and is a potential cause of many gastroduodenal diseases such as Peptic ulcer diseases, Non ulcer dyspepsia, MALT lymphoma and gastric cancer $(1,2,3)$. Invasive and noninvasive methods are available for the diagnosis of H.pylori infection. Obtaining biopsy through endoscopy is an invasive method, where biopsies are subjected to rapid urease test, culture, histopathology and DNA amplification, whereas urea breath test, serology and stool antigen test comes under non-invasive methods (4). Rapid urease test is the common diagnostic tool used for the detection of H.pylori infection. In Rapid urease test bacterial density plays a very important role. The density of H.pylori should be high for the RUT to be positive. If any agent leads to the reduction of bacterial load then there are high chances of getting false -negative results. To obtain a better results of rapid urease test, it is advised to go for more number of biopsies and also to increase the number of sites from the stomach (5). If the patients are on PPI, antibiotics or bismuth compounds, sensitivity of RUT is decreased $(6,7)$. There will be reduction in the number of bacteria when the patients are on antibiotics (8).The presence of drugs which are known to decrease the acid in the stomach, thereby increasing the $\mathrm{pH}$ affects the site of the stomach to be biopsied. Hence the site of the stomach from which biopsy is obtained plays an important role in determining the results of RUT (9). The main objective of this study was to determine the effect of PPI on the results of rapid urease test (PYLO DRY) and histopathology.

\section{Materials and Methods}

Biopsies were obtained from 35 dyspeptic patients, who underwent gastro intestinal endoscopy 2015 at Department of Gastroenterology, JSS Hospital Mysore from March 2015 to July2015. All patients had used PPI. Clinical symptoms at the time of presentation and diagnosis were noted with endoscopic findings. All adult patients of both genders, undergoing upper gastrointestinal endoscopy with features of peptic ulcer disease, Non ulcer dyspepsia and gastric carcinoma were included in this study. Patients with present or past history of gastric surgery or long term therapy with non-steroidal anti-inflammatory drugs were excluded from this study.An informed consent from all patients and approval from the institutional ethical committee were obtained before proceeding. 


\section{Biopsy Specimens:}

Two antral biopsies were obtained from each patient. Biopsy specimens were evaluated by Rapid urease test and histopathological examination.

\section{Rapid Urease Test:}

PYLO DRY (a commercially manufactured Rapid urease test from HALIFAX RESEARCH LABORATORY KOLKATA, INDIA) was performed according to the manufacturer's instructions. PYLO DRY consists of a dry filter paper containing urea, phenol red (a pH indicator), buffers and bacteriostatic agent in a sealed plastic slide. If the urease enzyme of H.pylori is present in an inserted tissue sample the resulting decomposition of urea causes the $\mathrm{pH}$ to raise and the colour of the dot turns from yellow to pink or red. Antral biopsy from each patient was placed into the test dots. After releasing the test the label was pressed over the test dot with the finger to squeeze the tissue fluid out of the specimens. The tissue fluid was absorbed by the filter paper. Results were monitored at room temperature after one hour and 24 hours. A positive result was defined as the colour change on the test from yellow to pink-magenta, and no colour change as negative for PYLO DRY

\section{Histopathological Examination for H.pylori Infection}

Antral biopsies were fixed in $10 \%$ formalin, before embedding in paraffin wax, the sections were stained with haematoxylin and eosin (H\&E) and Giemsa (in doubtful cases) for light microscopy to detect H.pylori infection. The presence of spiral organism on the slide was considered as positive for H.pylori infection. Histopathologist was kept blinded about the results of PYLO DRY

\section{Statistical Analysis}

Data was analysed using SPSS software. The sensitivity, specificity, positive predictive value and negative predictive value of PYLO DRY at one hour and 24 hours were calculated. The histopathologic findings were used as the gold standard for diagnosis of H.pylori infection.

\section{Results}

34 patients were recruited, of these 21 were males and 13 were females. The mean age was 46 years with a range of 21 to 90 years. The endoscopic findings were shown in Table 1. The most common endoscopic findings were Non ulcer dyspepsia, Gastric ulcer, duodenal ulcer and adenocarcinoma. Abdominal pain was present in 35\% (12), APD 26.4\% (9), Dyspepsia 8.8\% (3), GERD 8.8\% (3), and Hematemesis 5.8\% (2) (TABLE 1).

\section{Rapid Urease Test (PYLO DRY) and Histopathology}

PYLO DRY was positive in $17.6 \%$ (6) and $53 \%$ (18) when observed after one hour and 24 hours respectively. Among 8 patients who had positive histopathology for H.pylori, 3 patients had positive RUT at one hour and 5 patients had positive RUT at 24 hours. There were 12 Patients who had negative RUT at one hour and positive results at 24 hours.

\section{Comparison of Rapid urease test and Histopathology}

The sensitivity, specificity, positive predictive value (PPV) and negative predictive value (NPV) of PYLO DRY at one hour were 50\%, 82\%, 37.5\% and 88\% respectively. AT 24 hours they were $27 \%, 81 \%$, $62.5 \%$ and $50 \%$ respectively (TABLE 2 and 3 ).

Table 1: showing demographic data, symptoms, endoscopic findings, results of rapid urease test and histopathology

\begin{tabular}{|l|l|}
\hline Factors & Patients (\%) \\
\hline Gender & \\
Male & $21(61 \%)$ \\
Female & $13(38 \%)$ \\
\hline Age (year) +/-SD & \\
\hline Clinical symptoms & \\
Pain abdomen & $12(35 \%)$ \\
APD & $9(26.4)$ \\
Dyspepsia & $3(8.8 \%)$ \\
GERD & $3(8.8 \%)$ \\
Hematemesis & $2(5.8 \%)$ \\
Anorexia & $2(5.8 \%)$ \\
GI bleed & $2(5.8 \%)$ \\
Anaemia & $1(2.9 \%)$ \\
\hline Endoscopic findings & \\
Gastric ulcer & $8(23.5 \%)$ \\
\hline
\end{tabular}




\begin{tabular}{|c|l|}
\hline Duodenal ulcer & $8(23.5 \%)$ \\
Non ulcer & $16(47 \%)$ \\
Adenocarcinoma & $2(5.8 \%)$ \\
\hline Rapid urease test & \\
At one hour & $6(17 \%)$ \\
Positive & $28(82 \%)$ \\
Negative & \\
\hline After 24 hours & $18(52 \%)$ \\
Positive & $16(47 \%)$ \\
Negative & \\
\hline Histopathology & $8(23.5 \%)$ \\
Positive & $26(76.4 \%)$ \\
Negative & \\
\hline
\end{tabular}

Table2: The results of rapid urease test at one and 24 hours compared with histopathologic examinations

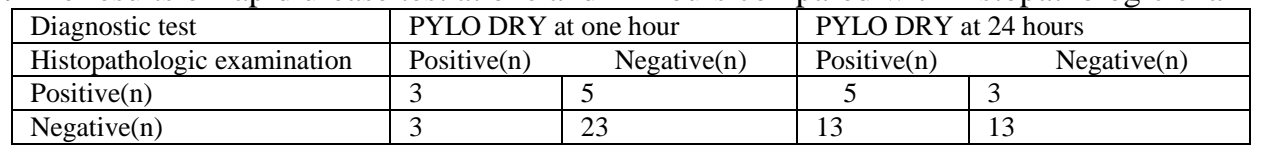

Table 3: The sensitivity, specificity, PPV and NPV of rapid urease test at one and 24 hours compared with histopathologic examinations

PPV $=$ Positive Predictive value

\begin{tabular}{|l|l|l|l|l|}
\hline Test & Sensitivity & Specificity & PPV & NPV \\
\hline Urease test at one hour & $50 \%$ & $82 \%$ & $37.5 \%$ & $88 \%$ \\
\hline Urease test at one 24 hours & $27 \%$ & $81 \%$ & $62.5 \%$ & $50 \%$ \\
\hline
\end{tabular}

NPV = Negative Predictive value

\section{Discussion}

Generally, PPIs are prescribed to dyspeptic patients for a specific duration, if the condition of the patient is not improving then they are advised to stop PPI before recruiting to endoscopy. According to previous studies, the recent usage of PPIs were known to reduce the sensitivity of diagnostic tests for H.pylori (10). In India the prevalence of H.pylori infection is around $80 \%$ (11). In our study the prevalence of H.pylori is less because patients had self-prescribed PPIs before upper GI endoscopy though the physicians advised to avoid PPIs. Previous studies suggest that, the density of H,pylori was reduced by PPIs and there by shifting their distribution (7), resulting in reduced sensitivity of several diagnostic tests available for H.pylori infection like RUT, Histopathology, culture, urea breath test, PCR and stool antigen test $(10,12)$. Our study shows that the sensitivity of PYLO DRY test at one hour in diagnosis of H.pylori infection was lower than the sensitivity at 24 hours, but specificity was more. 12 patients had negative results at 1 hour and showed positive results after 24 hours. Among these patients, 8 patients had positive histopathological findings. This indicates that the sensitivity of RUT increases at 24 hours and also increases the chances of getting false positive results. Earlier studies suggest that if density of H,pylori was less then RUT requires longer time to turn positive $(13,14)$. The load of H.pylori in the stomach can be reduced by PPIs. RUT may require longer time to detect H.pylori in patients who had PPI. In our study, we found that 28 patients had negative PYLO DRY test at 1 hour and negative histopathological examination for H.pylori infection. In contrast, 12 out of these 28 samples were positive at 24 hours with PYLODRY. PYLO DRY results obtained at 24 hours might be false positive as patients who were on PPIs might develop achlorhydria with subsequent superficial colonization by other urease producing organisms like Klebseilla or Proteus mirabilis $(15,16)$. These results might be false negative as histopathological examination was considered as gold standard. Chey WD et al. intheir study found that the sensitivity, specificity and Histopathologic examination was high in patients who were not on PPIs, whereas the sensitivity was reduced in patients who are on PPIs (10). Histopathologic examination may not be sensitive enough to analyse the results of PYLO DRY at 24 hours as true positives or false positives, in case of patients who were on PPI.

\section{Conclusion}

Consumption of PPI affects the results of rapid urease test as there high chances of ending up with false negative results. Hence it is very important to know weather PPI has been used or not before recruiting to endoscopy. Physicians should create an awareness against the self prescription of PPIs if the patient is recruited for endoscopy. if it is very difficult to avoid PPI, multiple biopsies from different parts of the stomach should be taken. In this study, 24 hours Rapid urease test yeilded higher sensitivity but lower specificity compared to RUT at 1 hour for diagnosis of H.pylori infection in patients who were on PPI. Further studies are required to determine the proper diagnostic method for H.pylori infection in patients who are on PPIs. 


\section{Acknowledgements}

This study was supported by grants from Department of Science and Technology, NewDelhi. The help provided by Dr H.P Nandeesh and Dr.Sunila is greatly appreciated.

\section{References}

[1] Manasa P Kumari, Sunila, Sumana MN, Nandeesh HP. The Histopathological study on Helicobacter pylori associated gastroduodenal diseases in a tertiary care hospital, Mysore. IJSR 2015; 4(7): 1973-1376

[2] Blaser MJ. Ecology of Helicobacter pylori in the human stomach. J Clin Invest1997;100:759-62.

[3] Blaser MJ. Perez-Perez GI, Kleanthous H, et al, Infection with Helicobacter pylori strains possessing cag A is associated with an increased risk of developing adenocarcinoma of the stomach. Cancer Res 1995; 55:2111-5.

[4] Vaira D,Gatta L, Ricci C et al. Review artice: diagnosis of Helicobacter pylori infection. Aliment Pharmacol Ther 2002; 16(Suppl 1); 16-23

[5] Lam SK. Talley NJ. Consensus conference on the management of Helicobacter pylori infection. J Gastroenterol Hepatol 1998.1:112

[6] Laine L Estrada R. Trujillo M, Fennerty MB. Effect of Proton pump inhibitor therapy on diagnostic test for Helicobacter pylori. Ann Intern Med 1998, 129: 5470550.

[7] Dickey W Kenny BD, McConnell JB. Effect of Proton pump inhibitors on the detection of Helicobacter pylori in gastric biopsies. Aliment Pharmacol Ther 1996, 10:289-293.

[8] Marshall BJ. Treatment strategies for Helicobacter pylori infection. Gastroenterol Clin North Am 1993, 22: 183-198.

[9] Stolte M. Bethke B. Elimination of Helicobacter pylori under treatment with omeprazole. Zekschrift Fur Gastroenterologie 1990, 28:271-274.

[10] Chey WD, Wong BC. American College of Gastroenterology guideline on the management of Helicobacter pylori infection, Am J Gastroenterol 2007;102:1808-25.

[11] Mhaskar RS, Ricardo I, Azliyati A, Laxminarayan R, Amol B, Santosh W, Boo K. Assessment of risk factors of helicobacter pylori infection and peptic ulcer disease. J Global Infect Dis 2013;5:60-7

[12] Graham DY, Opekun AR, Hammoud F, et al. Studies regarding the mechanism of falsenegative urea breath tests with proton pump inhibitors. Am J Gastroenterol 2003;98:1009-9.

[13] Kuo $\mathrm{CH}$, Wu DC, Lu CY, et al. The media of rapid urease test influence the diagnosis of Helicobacter pylori. Hepatogastroenterology 2002;49:1191-4.

[14] Marshall BJ, warren JR, Francis GJ, et al. Rapid urease test in the management of Campylobacter pyloridis-associated gastritis. Am J Gastroentrol 1987;82:200-10.

[15] Xia HX, KeaneCT, O’Morain CA. Preformed urease activity of Helicobacter pylori as determined by a viable cell count technique clinical implications. J Med Micobiol 1994;40:435-9.

[16] Midolo P, Marshall BJ. Accurate diagnosis of Helicobacter pylori. Urease tests. Gastroenterol Clin North Am 2000;29:871-8.

[17] D.S. Chan, Theory and implementation of multidimensional discrete systems for signal processing, doctoral diss., Massachusetts Institute of Technology, Cambridge, MA, 1978. (8)

Note that thesis title is set in italics and the university that granted the degree is listed along with location information

Proceedings Papers:

[18] W.J. Book, Modelling design and control of flexible manipulator arms: A tutorial review, Proc. 29th IEEE Conf. on Decision and Control, San Francisco, CA, 1990, 500-506 (8) 